\title{
Evaluación de Extractos Vegetales y Bioinsecticidas Sobre Poblaciones de Spodoptera frugiperda y Elasmopalpus lignosellus en Maíz
}

\author{
Ing. Agrp. Teddy Landívar Ortiz \\ Ing. Agr. Eduardo Colina Navarrete Mg. Sc. \\ Ing. Ind. Carlos Castro Arteaga Mg. Sc. \\ Ing. Agr. Danilo Santana Aragoné \\ Ing. Agr. Guillermo García Vásquez Mg. Sc. \\ Ing. Agr. Oscar Mora Castro MBA \\ Lcda. Martha Uvidia Vélez MIS \\ Universidad Técnica de Babahoyo, Ecuador \\ Ing. Agr. Miguel Goyes Cabezas MBA \\ Unidad Nacional de Almacenamiento-UNA
}

doi: 10.19044/esj.2017.v13n21p238 URL:http://dx.doi.org/10.19044/esj.2017.v13n21p238

\begin{abstract}
The research was carried out on the Property "El Cartan" that is located in Km 4 way at Puebloviejo-Ventanas, county of Los Ríos. A test was conducted on the hybrid corn DK-1040. This test measures the response of the larvae of insects Spodoptera frugiperda and Elasmopalpus lignosellus to the application of dose of biological and organic insecticides. Also, the most appropriate dose in products was determined with an economic analysis. The experimental design used was random complete blocks with seven treatments and three replicates. The variables evaluated are: percentage of larvae control, number of larvae per plant, corn damage, and yield for hectare. The evaluated variables were subjected to the analysis of variance, and Tukey test was applied to $95 \%$ probability. The analyzed results were determined to apply Neem (Azadirachtina indica) in dose of 1,0 $\mathrm{L} \mathrm{ha}^{-1}$. It diminishes the populations of S. frugiperda and E. lignosellus in relationship to the other active matters applied in the test. Higher doses lead to migration of pests to nearby hosts. The highest yield per hectare was found in the Neem 1.0 L ha-1 treatment with $8,940 \mathrm{~kg}$ ha-1.
\end{abstract}

Keywords: Sustainable agriculture, Insects, Control, Individuals, Yield 


\section{Resumen}

La investigación fue realizada en la Finca "El Cartan", ubicada en el Km 4 vía Puebloviejo-Ventanas, Provincia de Los Ríos. Se estableció un ensayo en el maíz híbrido DK-1040, midiendo la respuesta de las larvas de los insectos Spodoptera frugiperda y Elasmopalpus lignosellus a la aplicación de dosis de insecticidas biológicos y orgánicos, además se determinó la dosis más adecuada de productos con un análisis económico. El diseño experimental utilizado fue Bloques completos al azar con siete tratamientos y tres repeticiones. Las variables evaluadas fueron: porcentaje de control de larvas, número de larvas por planta, daño en mazorca y rendimiento por hectárea. Las variables evaluadas fueron sometidas al análisis de variancia, y se aplicó la prueba de Tukey al $95 \%$ de probabilidad. Analizados los resultados se determinó que aplicar Neem (Azadirachtina indica) en dosis de 1,0 $\mathrm{L} \mathrm{ha}^{-1}$, disminuye las poblaciones de $S$. frugiperda y E. lignosellus con relación a las otras materias activas aplicadas en el ensayo. Dosis mayores, ocasionan migración de las plagas hacia hospederos cercanos. El mayor rendimiento por hectárea se encontró en el tratamiento Neem 1,0 $\mathrm{L} \mathrm{ha}^{-1}$ con $8940 \mathrm{~kg} \mathrm{ha}^{-1}$.

Palabras Claves: Agricultura Sostenible, Insectos, Control, Individuos, Rendimiento

\section{Introducción}

El maíz (Zea mays), es por hoy uno de los productos agrícolas más importante de la economía Ecuatoriana y mundial, esto debido a que casi las tres cuartas partes de la producción total proviene de unidades familiares campesinas, la mayorías de ella de economías de subsistencia, teniendo una elevada incidencia social.

El mundo siembra anualmente 129,2 millones de hectáreas aproximadamente, alcanzado una producción de alrededor de 477,4 millones de toneladas métricas. En Ecuador este cultivo es el segundo grano más importante en la alimentación humana después del arroz, empleando alrededor de 40000 personas en mano de obra, correspondiente el $11 \%$ de la población económicamente activa dedicada a la agricultura. La siembra de 320000 hectáreas, se producen sobre todo en las provincias de Los Ríos, Guayas y Manabí con el 60 \% del área toral nacional (INEC-SINAGAP, 2014).

Para Quijije (1996) el gusano cogollero se origina en América y el caribe, donde se encuentra distribuida en toda la América tropical, desde Estados Unidos, Centro América y Suramérica. El cogollero (Spodoptera frugiperda) constituye la plaga más importante del cultivo de maíz, las 
pérdidas que esta insecto ocasiona son cuantiosas, pudiendo reducir los rendimientos hasta el $80 \%$ del tejido foliar. Este problema se da por cuanto durante mucho tiempo, para reducir los efectos nocivos de S. frugiperda los agricultores realizan el control pasado el momento crítico de la plaga y la etapa fenológica más apropiada del cultivo o después que los daños son irreversibles. El INIAP (1992) menciona que un control adecuado debe realizarse, cuando la población que sobrepasa el umbral económico llegue al $5 \%$ de plantas atacadas, ya que Navarro (2000) señala que en la etapa de crecimiento, las larvas de cogollero se alimentan del cogollo, consumen principalmente las hojas más tiernas y que influyen sobre el rendimiento, reduciendo el área fotosintética, realizando siempre el control con productos inhibidores de quitina.

El gusano barrenador Elasmopalpus lignosellus ha sido reportado en la mayoría de los países de Sudamérica, al igual que parte de centro América y la región oeste de los Estados Unidos (Betancourt, 1999). El gusano ejército (E. lignosellus) es un insecto que ataca una gran cantidad de plantas cultivadas entre las que se destacan: caña de azúcar, soya, arroz y malezas. Esta larva tiende a alimentarse bajo la superficie del suelo, afectando el cuello y la porción subterránea del tallo. En otros casos taladran en sentido transversal las hojas en desarrollo, lo que lleva a que estas cuando crecen se visualicen perforaciones, siendo también una plaga errática que varía su incidencia sustancialmente de una año a otro. La severidad de los daños está relacionada con las condiciones climáticas, los periodos de sequía son los mejores para el desarrollo del insecto (Bentancourt \& Scatoni, 1999; Paliz \& Mendoza, 1985).

La manera eficaz de controlar estos insectos es mediante la combinación adecuada de los métodos cultural, químicos, y biológicos. El control cultural consiste en el control de malezas, siembras oportunas, arrancar y quemar las plantas atacadas. Los productos químicos que se aplican al momento de la siembra como a la semilla, resultan muy poco eficaces. Para el control biológico se han encontrado parásitos de larvas y pupas de estos insectos, que corresponden a las familias: Tachiniidae, Braconidae e Ichneumonidae. Para reducir estos problemas se procura la implementación de sistemas agrícolas sostenibles, basados en el conocimiento de las relaciones entre: los cultivos, el ambiente y los organismo presentes en el campo (Monzón, 2000).

Diferentes aspectos deberán ser mejorados para elevar los rendimientos es el manejo integrado de plagas, que constituye uno de los más importantes y apremiantes. El CIAT (1998) manifiesta que las aspersiones de insecticidas, deben realizarse cuando el nivel de daño y población de la plaga alcancen el umbral económico. En maíz para el control de plagas ha cambiado el sistema calendarizado por los cebos, cuando los 
daños sobrepasan el $50 \%$ y aspersiones al follaje cuando son menores. Quijije (1996) sostiene que la creciente demanda mundial del cultivo, hizo que los agricultores realizaran una protección total del cultivo a base de aplicaciones preventivas de insecticidas. A partir de entonces, se creó un nuevo problema fitosanitario, las poblaciones de las plagas aumentaron drásticamente.

En la actualidad el uso de microorganismos con potencialidad para ser usados en la lucha contra insectos, ha aumentado considerablemente, siendo este afectado cuando se aplica en los primeros estadios larvales (FAO, 1992). Según Roberts (1999), el uso de agentes organismos en la agricultura, se viene dando desde muchos años, debido a la tendencia moderna de disminuir el uso de plaguicidas químicos debido, a la resistencia de las plagas a los mismos. Khamby et al. (1991) por su parte en investigaciones realizadas sobre las dos vertientes que tiene la agricultura sobre los insecticidas botánicos, encontraron que la agricultura de subsistencia y la agricultura sostenible, buscan tener alternativas de control que permitan tener armas para disminuir la incidencia de plagas, aprovechando por completo los recursos agrícolas, mediante el reciclaje de las materias primas.

Se conoce como control bioinsecticida o insecticida botánico, a aquel que produce una disminución en la población de una plaga, sin causar problemas en el medio ambiente o contaminar el mismo (Suquilanda, 2002). Como alternativa la práctica de utilización de bioinsecticidas y extractos naturales ha originado una nueva tendencia sobre el control y manejo de insectos plagas en muchos cultivos.

Los objetivos planteados en este investigación fueron: determinar el efecto de los extractos vegetales y tres bioinsecticidas sobre las poblaciones de S. frugiperda y Elasmopalpus lignosellus, establecer el control más eficientes para el control de los insectos en campo y analizar económicamente los tratamientos evaluados.

\section{Materiales y Métodos}

La investigación se realizó en la Finca "El Cartan" ubicada en el Km 4 de la vía Puebloviejo-Ventanas. Coordenada geográfica 79³2' de longitud oeste y $01^{\circ} 32^{\prime}$ de latitud sur, altura de $32 \mathrm{msnm}$, temperatura media anual $25,7^{\circ} \mathrm{C}$, precipitación anual 1925 , humedad relativa $80 \%$ (INAHMI, 2016). El suelo es de origen aluvial, con topografía plana, textura franco arcillosa, drenaje natural. Se utilizó el híbrido DK-1040 (Ecuaquimica, 2014).

Se empleó un análisis de varianza para determinar las significancias entre los tratamientos y comparación de medias de tratamientos con Tukey al $95 \%$ de probabilidades. Las variables evaluadas fueron: número de larvas por planta, porcentaje de control de insecticidas, mortalidad de insectos a las 24, 48, 72 y 96 horas después de la aplicación, días a la floración, días a 
maduración fisiológica, daño en mazorca, rendimiento por hectárea y análisis económico.

\section{Tratamientos}

Los tratamientos evaluados fueron obtenidos de los insecticidas biológicos: Bacillus thurigiensis (New BT), Trichoderma spp. (Custom GP), Azadirachtina indica (Neem X), Crisantemun spp. (Phyrisect), Ácidos Orgánicos (NP-600), Cypermetrina (Cyperpac) y un testigo sin aplicación.

Tabla 1. Tratamientos

\begin{tabular}{|c|c|c|c|}
\hline & Ingrediente activo & $\begin{array}{c}\text { Concentración } \\
\mathrm{g} / \mathrm{L}\end{array}$ & $\begin{array}{c}\text { Dosis } \\
\mathrm{cc} / \mathrm{ha}\end{array}$ \\
\hline T1 & Azadirachtina indica & 300 & 500 \\
\hline T2 & Crisantemun spp & 50 & 1000 \\
\hline T3 & Trichoderma spp. & $1 \times 10^{8}$ & 500 \\
\hline T4 & Bacillus thurigiensis & $1 \times 10$ & 500 \\
\hline T5 & Ácidos Orgánicos & 850 & 1000 \\
\hline T6 & Cypermetrina & 200 & 300 \\
\hline T7 & \multicolumn{2}{|c|}{ Sin aplicación } \\
\hline
\end{tabular}

Para el trabajo de investigación se utilizó el diseño de bloques completos al azar, en tres repeticiones. Las aplicaciones de los insecticidas se realizaron cuando el umbral de daño se ubicó en el $10 \%$. Previo al establecimiento del ensayo se tomaron muestras de suelos, para proceder al análisis físico y químico; determinando así la cantidad de nutrientes existentes. El análisis de concentración se realizó en los laboratorios de microbiología del INIAP.

El terreno fue preparado con un pase de arado y dos pases de rastra liviana cruzados, sembrando en forma manual colocando 1 semilla por sitio a la distancia de 0,80 m entre hileras y 0,20 m entre plantas. Para el control de malezas se aplicó pendimetalin en dosis de $3 \mathrm{~L} \mathrm{ha}^{-1}$ y atrazina $1 \mathrm{~kg} \mathrm{ha}^{-1}$ después de la siembra. a los 35 y 55 días después de la siembra se controló las malezas de forma manual con rabón.

No se reportó ataque de enfermedades en el cultivo, por lo cual no se realizó aplicaciones de fungicidas. El cultivo fue realizado en la época lluviosa 2016, con lo cual no se realizó aportes hídricos al ensayo.

Las dosis de fertilización química se aplicaron según los resultados del análisis de suelo, determinando $150 \mathrm{~kg} \mathrm{ha}^{-1}$ (Nitrógeno), $25 \mathrm{~kg} \mathrm{ha}^{-1}$ (Fósforo), $75 \mathrm{~kg} \mathrm{ha}^{-1}$ (Potasio), $14 \mathrm{~kg} \mathrm{ha}^{-1}$ (Azufre) y 1,3 kg ha'-1 (Boro). Los productos fosforados y potásicos fueron colocados el $50 \%$ a la siembra y el restante a 20 días después de la siembra. El nitrógeno y el azufre fueron colocados el $20 \%$ a la siembra, $50 \%$ a 20 días después de la siembra y el 30 \% 15 días después de esta. El Boro se aplicó foliar con Metalosato Boro en dosis de $0,3 \mathrm{~L} \mathrm{ha}^{-1}$, mensualmente hasta la emergencia de la flora masculina. 
La cosecha fue manual, cuando las mazorcas tuvieron una humedad del 22 $\%$.

El número de larvas por planta se contó en 10 plantas al azar por tratamiento, tanto de S. frujiperda como E. lignosellus, cinco días antes de aplicar aplicación y por cada aplicación de insecticidas. Los valores del porcentaje de control de insecticidas, se hizo restando el número de larvas vivas 5 días antes de la aplicación de producto, con el número de larvas vivas a las 96 horas después de la misma.

Los daños en las mazorca fueron tomados al momento de cosecha contando en 10 plantas al azar, cuantas presentaron daño en su interior por ataque de las larvas, basado en la siguiente escala: 1= No hay daño, 2= Ataque no mayor de $1 \mathrm{~cm}$ de perforación, 3= Ataque mayor a $2 \mathrm{~cm}$ de perforación, 4= Hileras de granos trazadas y mordidas, 5= Más del 50\% de mazorca trazada o mordida.

El número de días a la floración fue evaluado desde la fecha de siembra hasta cuando el $50 \%$ del total de las plantas de cada parcela experimental, presentaron floración masculina. De la misma manera los días a maduración fisiológica fueron tomados cuando el cultivo tuvo un $100 \%$ de secado de grano y las vellosidades de la punta de mazorca, estuvieron de color negro. El rendimiento por hectárea fue evaluado en cada unidad experimental, recolectando la cosecha del área útil y uniformizando al 13\% de humedad. El análisis económico se basó en los costos de producción e ingresos.

\section{Resultados}

Número de larvas vivas por planta de Spodoptera frugiperda y Elasmoplapus lignosellus, en las evaluaciones realizadas después de la aplicación de insecticidas.

\section{Spodoptera frugiperda}

El número de larvas por planta, dio alta significancia estadística en las evaluaciones realizadas (Tabla 2). Las evaluaciones realizadas 5 días antes de la aplicación demostraron que los tratamientos Azadirachtina indica, Bacillus thurigiensis y testigo absoluto tuvieron el mayor número de larvas por planta (1,0 larva/planta), siendo estadísticamente superiores a los demás. El menor promedio se obtuvo en los tratamientos Crisantemun spp, Trichoderma spp., Ácidos Orgánicos y Cypermetina con 0,9 larvas/planta. El coeficiente de variación fue $3,51 \%$.

Los promedios a 24 y 48 horas después de la aplicación, dieron en el testigo absoluto superioridad estadística sobre los demás tratamientos con 0,5 larvas vivas/planta. Los menores promedios se presentaron aplicando 
Azadirachtina indica, Bacillus thurigiensis, Crisantemun spp, Ácidos Orgánicos y Cypermetina, con 0 larvas vivas/planta (C.V. 2,67 \%).

A 72 y 96 horas el testigo absoluto fue estadísticamente superior a los demás tratamientos con 0,5 larvas vivas/planta. El menor promedio se obtuvo en los tratamientos Azadirachtina indica, Bacillus thurigiensis, Crisantemun spp. y Ácidos Orgánicos, con 0 larvas vivas/planta (C.V. 2,46 $\%)$.

\section{Elasmoplapus lignosellus}

Los valores alcanzaron alta significancia estadística al $5 \%$ de probabilidad para todas las evaluaciones realizadas (Tabla 2). Cinco días antes de la primera aplicación el mayor valor de número de larvas $(0,6)$ se obtuvo en el tratamiento Ácidos Orgánicos, siendo estadísticamente superior a los demás tratamientos. El menor promedio se encontró en los tratamientos Trichoderma spp., Bacillus thurigiensis y testigo absoluto (0,1 larvas/plata). El coeficiente de variación fue 1,69\%.

Los promedios 24 horas después de la aplicación con Azadirachtina indica y Ácidos Orgánicos (0,2 larvas planta) fueron estadísticamente superiores a los demás tratamientos. Menor valor se reportó en Trichoderma spp., Crisantemun spp., Bacillus thurigiensis, Testigo absoluto $\mathrm{y}$ Cypermetrina, con 0,1 larvas vivas/planta. El coeficiente de variación fue $2,81 \%$.

Las observaciones a 48, 72 y 96 horas después, expresaron que Trichoderma spp. fue estadísticamente superior a los demás tratamientos con 0,2 larva viva/planta. Los menores promedios se encontraron en los tratamientos Azadirachtina indica, Crisantemun spp., Bacillus thurigiensis, testigo absoluto y Cypermetrina, con 0 larvas vivas/planta. El coeficiente de variación fue $1,07 \%$, para las observaciones.

Tabla 2. Número de larvas vivas por planta de S. frugiperda y E. lignosellus, en las evaluaciones realizadas después de la aplicación de los insecticidas. Los Ríos, 2016.

\begin{tabular}{|c|c|c|c|c|c|c|c|c|c|c|}
\hline \multirow{3}{*}{ Tratamiento } & \multirow{2}{*}{\multicolumn{2}{|c|}{$\begin{array}{c}\text { Larvas } \\
\text { vivas } 5 \\
\text { días antes } \\
\text { de } \\
\text { aplicació } \\
\text { n } \\
\end{array}$}} & \multicolumn{4}{|c|}{$\begin{array}{l}\text { Larvas vivas } \\
\text { S. Frugiperda }\end{array}$} & \multicolumn{4}{|c|}{$\begin{array}{l}\text { Larvas vivas } \\
\text { E. lignosellus }\end{array}$} \\
\hline & & & $\begin{array}{c}24 \\
\text { hda }\end{array}$ & $\begin{array}{c}48 \\
\text { hda }\end{array}$ & $\begin{array}{c}72 \\
\text { hda }\end{array}$ & $\begin{array}{c}96 \\
\text { hda }\end{array}$ & $\begin{array}{c}24 \\
\text { hda }\end{array}$ & $\begin{array}{c}48 \\
\text { hda }\end{array}$ & $\begin{array}{r}72 \\
\text { hda }\end{array}$ & $\begin{array}{c}96 \\
\text { hda }\end{array}$ \\
\hline & & $\mathrm{El}$ & & & Prim & $\mathrm{a} A \mathrm{p}$ & icaci & & & \\
\hline Azadirachtina indica & $\begin{array}{c}0,9 \\
b\end{array}$ & $\begin{array}{c}1,0 \\
\mathrm{a}\end{array}$ & $0,1 \mathrm{c}$ & $0,1 \mathrm{c}$ & $0,0 \mathrm{~d}$ & $\begin{array}{c}0,0 \\
d\end{array}$ & $\begin{array}{c}0,2 \\
\mathrm{a}\end{array}$ & $\begin{array}{c}0,0 \\
\mathrm{c}\end{array}$ & $\begin{array}{c}0,0 \\
\mathrm{c}\end{array}$ & $0,0 \mathrm{c}$ \\
\hline Crisantemun spp & $\begin{array}{c}0,9 \\
b\end{array}$ & $\begin{array}{c}0,9 \\
b\end{array}$ & $0,1 \mathrm{c}$ & $0,1 \mathrm{c}$ & $0,0 \mathrm{~d}$ & $\begin{array}{c}0,0 \\
d\end{array}$ & $\begin{array}{c}0,1 \\
\mathrm{~b}\end{array}$ & $\begin{array}{c}0,0 \\
\mathrm{c}\end{array}$ & $\begin{array}{c}0,0 \\
\mathrm{c}\end{array}$ & $0,0 \mathrm{c}$ \\
\hline Trichoderma spp. & $\begin{array}{c}0,9 \\
b\end{array}$ & $\begin{array}{c}0,9 \\
b\end{array}$ & $0,3 \mathrm{~b}$ & $0,3 \mathrm{~b}$ & $0,3 \mathrm{~b}$ & $\begin{array}{c}0,3 \\
b\end{array}$ & $\begin{array}{c}0,1 \\
b\end{array}$ & $\begin{array}{c}0,2 \\
\mathrm{a}\end{array}$ & $\begin{array}{c}0,2 \\
\mathrm{a}\end{array}$ & $0,2 \mathrm{a}$ \\
\hline
\end{tabular}




\begin{tabular}{|c|c|c|c|c|c|c|c|c|c|c|}
\hline Bacillus thurigiensis & $\begin{array}{c}1,0 \\
\mathrm{a}\end{array}$ & $\begin{array}{c}1,0 \\
\mathrm{a}\end{array}$ & $0,1 \mathrm{c}$ & $0,1 \mathrm{c}$ & $0,0 \mathrm{~d}$ & $\begin{array}{c}0,0 \\
\mathrm{~d}\end{array}$ & $\begin{array}{c}0,1 \\
b\end{array}$ & $\begin{array}{c}0,0 \\
\mathrm{c}\end{array}$ & $\begin{array}{c}0,0 \\
\mathrm{c}\end{array}$ & $0,0 \mathrm{c}$ \\
\hline Ácidos Orgánicos & $\begin{array}{c}0,8 \\
\mathrm{c}\end{array}$ & $\begin{array}{c}0,9 \\
b\end{array}$ & $0,1 \mathrm{c}$ & $0,1 \mathrm{c}$ & $0,0 \mathrm{~d}$ & $\begin{array}{c}0,0 \\
\mathrm{~d}\end{array}$ & $\begin{array}{c}0,2 \\
\mathrm{a}\end{array}$ & $\begin{array}{c}0,1 \\
b\end{array}$ & $\begin{array}{c}0,1 \\
b\end{array}$ & $0,1 \mathrm{~b}$ \\
\hline Cypermetrina & $\begin{array}{c}0,9 \\
b\end{array}$ & $\begin{array}{c}0,9 \\
b\end{array}$ & $0,1 \mathrm{c}$ & $0,1 \mathrm{c}$ & $0,1 \mathrm{c}$ & $\begin{array}{c}0,1 \\
\mathrm{c}\end{array}$ & $\begin{array}{c}0,1 \\
b\end{array}$ & $\begin{array}{c}0,0 \\
\mathrm{c}\end{array}$ & $\begin{array}{c}0,0 \\
\mathrm{c}\end{array}$ & $0,0 \mathrm{c}$ \\
\hline Testiq & $\begin{array}{c}0,9 \\
b\end{array}$ & $\begin{array}{c}1,0 \\
\mathrm{a}\end{array}$ & $0,5 \mathrm{a}$ & $0,5 \mathrm{a}$ & $0,5 \mathrm{a}$ & $\begin{array}{c}0,5 \\
\mathrm{a}\end{array}$ & $\begin{array}{c}0,0 \\
\mathrm{c}\end{array}$ & $\begin{array}{c}0,0 \\
\mathrm{c}\end{array}$ & $\begin{array}{c}0,0 \\
\mathrm{c}\end{array}$ & $0,0 \mathrm{c}$ \\
\hline Pron & 0,9 & 0,9 & 0,2 & 0,2 & 0,1 & 0,1 & 0,1 & 0,0 & 0,0 & 0, \\
\hline $\begin{array}{l}\text { Significancia } \\
\text { Estadísticas }\end{array}$ & $* *$ & $* *$ & $* *$ & $* *$ & * & $* *$ & $* *$ & $* *$ & $* *$ & ** \\
\hline C.V. $\%$ & $\begin{array}{c}3.5 \\
1\end{array}$ & $\begin{array}{c}1.6 \\
9 \\
\end{array}$ & 2.67 & 2.67 & 2.46 & $\begin{array}{c}2.4 \\
6 \\
\end{array}$ & $\begin{array}{c}2.8 \\
1 \\
\end{array}$ & $\begin{array}{c}1.0 \\
7 \\
\end{array}$ & $\begin{array}{c}1.0 \\
7 \\
\end{array}$ & 1.07 \\
\hline
\end{tabular}

h.d.a.: horas después de la aplicación.

Medias con una letra en común no son significativamente diferentes Tukey $(\mathrm{p} \leq 0,05)$. $\sqrt{ }+1$.

Para análisis estadístico de los valores originales se transformó a

Los datos demuestran que las aplicaciones realizadas, influyen sobre la dinámica de las larvas, ya que a partir de las 72 horas después de la aplicación, la mortalidad de larvas es más estable, ya que la mayoría insecticidas aplicados actúan por efecto de contacto o volteo (Ecuaquimica, 2010), además de provocar que las larvas no se alimenten, lo que ocasiona un lento control (Biotecdor, 2010), causando parálisis y luego muerte. El porcentaje de control, es mayor en productos que provienen de síntesis botánica sobre todo el Neem, el cual se ha reportado para el control de más de 150 especies de insectos, especialmente lepidópteros (INIAP, 2010).

Porcentaje de control de insecticidas aplicados sobre larvas de Spodoptera frugiperda y Elasmoplapus lignosellus

Los porcentajes de la mortalidad de larvas después de la aplicación de los tratamientos estudiados, no alcanzaron significancia estadística (Tabla $3)$.

Los promedios indican que la mejor eficiencia en control de las larvas de $S$. frugiperda se encuentra aplicando Azadirachtina indica, Crisantemun spp, Bacillus thurigiensis y Ácidos Orgánicos con (100\%), con menor control en el tetsigo sin aplicación.

La menor eficiencia de control en E. lignosellus se relaciona con Trichoderma spp. (77,8\%), siendo mayor el control con la aplicación de Azadirachtina indica, Crisantemun spp, Bacillus thurigiensis, Cypermetrina y en el Testigo absoluto. 
Tabla 3. Porcentaje de control de larvas de S. frugiperda y E. lignosellus, con aplicación de insecticidas botánicos y biológicos. Los Ríos, 2016.

\section{Porcentaje de Control}

Tratamiento

\begin{tabular}{ccc}
\hline & S. frugiperda & E. lignosellus \\
Azadirachtina indica & $100 \mathrm{a}$ & $100 \mathrm{a}$ \\
Crisantemun spp & $100 \mathrm{a}$ & $100 \mathrm{a}$ \\
Trichoderma spp. & $66,7 \mathrm{c}$ & $77,8 \mathrm{c}$ \\
Bacillus thurigiensis & $100 \mathrm{a}$ & $100 \mathrm{a}$ \\
Ácidos Orgánicos & $100 \mathrm{a}$ & $88,9 \mathrm{~b}$ \\
Cypermetrina & $89,9 \mathrm{~b}$ & $100 \mathrm{a}$ \\
Testigo absoluto & $44,4 \mathrm{~d}$ & $100 \mathrm{a}$ \\
\hline Promedios & 85,7 & 95,2 \\
Significancia Estadísticas & $* *$ & $* *$ \\
C.V. \% & 12.21 & 7,68 \\
\hline
\end{tabular}

h.d.a.: horas después de la aplicación.

Medias con una letra en común no son significativamente diferentes Tukey $(\mathrm{p} \leq 0,05)$.

Los análisis de estadística determinaron que las aplicaciones de Azadirachtina indica, Crisantemun spp. y Bacillus thurigiensis, lograron disminución de las poblaciones de los insectos en el $100 \%$ los casos, siendo menos eficiente en el control las cepas de Trichoderma spp. Esto se explica por las apreciaciones de Negrete y Morales (2003), quienes exponen que la aplicación de insecticidas botánicos y biológicos, lo que busca es disminuir la población de una plaga, sin causar un desequilibrio en el medio ambiente o contaminación del mismo, ocasionando mortalidades superiores al $80 \%$ de individuos.

Ademásel uso de plaguicidas provoca efectos negativos sobre el suelo, agua y ambiente. La reducción de estos efectos disminuirá con la implementación de sistemas agrícolas sostenibles, basados en el conocimiento de las relaciones entre: los cultivos, el ambiente y los organism presentes en le campo. Una de esas alternativas es el uso de organismos entomopatógenos e insecticidas botánicos (Monzón, 2000).

\section{Daño de mazorcas}

En la tabla 4, se presentan los promedios del daño en mazorcas de los tratamientos estudiados. El análisis de varianza no determinó significancia estadística al $95 \%$ de probabilidad (CV 7,12 y 4,23\%).

En las mazorcas evaluadas se encontraron larvas de Spodoptera frugiperda y Elasmoplapus lignosellus, lo que indican que bajo ciertas condiciones las larvas pueden alimentarse de otros órganos de las plantas como lo indica Ortega (1987), sin embargo los promedios de daño son bajos 
$(2,67$ y $2,52 \%$, en su orden), lo que no repercute en la producción del cultivo.

Tabla 4. Daño de mazorca, con la aplicación de extractos vegetales y bioinsecticidas. Los Ríos, 2016.

Porcentaje de Control

Tratamiento

\begin{tabular}{ccc}
\hline & S. frugiperda & E. lignosellus \\
Azadirachtina indica & 2,67 & 2,33 \\
Crisantemun spp & 3,00 & 2,33 \\
Trichoderma spp. & 2,67 & 2,67 \\
Bacillus thurigiensis & 3,00 & 2,33 \\
Ácidos Orgánicos & 2,00 & 2,33 \\
Cypermetrina & 2,33 & 2,67 \\
Testigo absoluto & 3,00 & 3,00 \\
\hline Promedios & 2,67 & 2,52 \\
Significancia Estadísticas & $* *$ & $* *$ \\
C.V. \% & 7,12 & 4,23 \\
\hline
\end{tabular}

h.d.a.: horas después de la aplicación.

Medias con una letra en común no son significativamente diferentes Tukey $(\mathrm{p} \leq 0,05)$.

Las investigaciones demuestran que una de las principales causas de pérdidas de los rendimientos del maíz, son los insectos de mazorca, que pueden llegar a ser considerables. Sin embargo, Guzmán y Alonso (2001) e Hilje (1993), consideran que en la mayoría de los casos, un cultivo limpio, vigoroso y con controles adecuados de insectos, difícilmente constituya un hábitat favorable para organismos dañinos, dentro de los principales especies mencionan a Spodoptera frugiperda y Elasmoplapus lignosellus.

\section{Rendimiento por hectárea}

El análisis de varianza alcanzó significancia estadística al 5\% de probabilidad. En la Tabla Cuadro 5, estos valores son presentados (CV 2,08 $\%)$.

Los promedios encontrados las plantas tratatadas con Azadirachtina indica $\left(8940,3 \mathrm{~kg} \mathrm{ha}^{-1}\right)$ para el control de insectos, presentaron el mayor rendimiento, estadísticamente superiores a los demás tratamientos. La menor producción se obtuvo en el testigo absoluto sin control de plagas $(3831,2 \mathrm{~kg}$ $\left.\mathrm{ha}^{-1}\right)$.

Tabla 5. Rendimiento por hectárea, con la aplicación de extractos vegetales y bioinsecticidas. Los Ríos, 2016.

\begin{tabular}{cc}
\hline Tratamiento & $\mathrm{Kg} \mathrm{ha}^{-1}$ \\
\hline Azadirachtina indica & $8940,3 \mathrm{a}$ \\
Crisantemun spp & $8010,4 \mathrm{~b}$ \\
Trichoderma spp. & $6645,2 \mathrm{~d}$ \\
Bacillus thurigiensis & $6126,1 \mathrm{e}$ \\
Ácidos Orgánicos & $7530,4 \mathrm{c}$
\end{tabular}




\begin{tabular}{cc} 
Cypermetrina & $6000,7 \mathrm{f}$ \\
Testigo absoluto & $3831,2 \mathrm{~g}$ \\
\hline Promedios & 6869,2 \\
Significancia Estadísticas & $* *$ \\
C.V. $\%$ & 2,08 \\
\hline
\end{tabular}

diferentes

Medias con una letra en común no son significativamente Tukey $(\mathrm{p} \leq 0,05)$.

Un control rápido y oportuno basado en un umbral económico de daño, evita las pérdidas en los cultivos, logrando así lograr un mayor rendimiento en peso de grano, sobre todo con el uso de insecticidas de bajo impacto ambiental, coincidiendo con lo ha manifestado por INIAP (1992). Sin embargo las aspersiones al follaje deben hacerse con umbrales de daño menores, como lo afirma el CIAT (1998). En la actualidad el uso de microorganismos con potencialidad para ser usados en la lucha contra insectos, ha aumentado considerablemente, siendo este afectado cuando se aplica en los primeros estadios larvales (FAO, 1992).

\section{Análisis económico}

La Tabla 6 muestra los valores referentes al análisis económico realizado a los tratamientos estudiados.

Se encontró que las plantas tratadas con Azadirachtina indica, dieron la mayor utilidad $\$ 1576,94$ dólares; mientras el menor ingreso lo tuvo el testigo absoluto sin control de plagas $(\$ 569,99)$.

Tabla 5. Análisis económico, con la aplicación de extractos vegetales y bioinsecticidas. Los

Ríos, 2016.

\begin{tabular}{|c|c|c|c|c|c|c|}
\hline Tratamiento & $\mathrm{Kg} \mathrm{ha}^{-1}$ & $\begin{array}{c}\text { Ingresos } \\
\$\end{array}$ & $\begin{array}{c}\text { Egresos } \\
\$\end{array}$ & $\begin{array}{c}\text { Utilidad } \\
\$\end{array}$ & $\mathrm{~B} / \mathrm{C}$ & $\mathrm{TR} \%$ \\
\hline Azadirachtina indica & 8940,3 & 2503,284 & 1055,91 & 1447,37 & 2,37 & 57,82 \\
\hline Crisantemun spp & 8010,4 & 2242,912 & 1012,99 & 1229,92 & 2,21 & 54,84 \\
\hline Trichoderma spp. & 6645,2 & 1860,656 & 961,92 & 898,74 & 1,93 & 48,30 \\
\hline Bacillus thurigiensis & 6126,1 & 1715,308 & 929,08 & 786,23 & 1,85 & 45,84 \\
\hline Ácidos Orgánicos & 7530,4 & 2108,512 & 1032,87 & 1075,64 & 2,04 & 51,01 \\
\hline Cypermetrina & 6000,7 & 1680,196 & 913,56 & 766,64 & 1,84 & 45,63 \\
\hline Testigo absoluto & 3831,2 & 1072,736 & 791,09 & 281,65 & 1,36 & 26,25 \\
\hline
\end{tabular}

\section{Conclusiones}

La aplicación de extractos de botánicos, en un programa de manejo de insectos plaga, disminuye la incidencia de larvas en porcentajes adecuados de control. El producto con Azadirachtina indica en dosis de 1,0 $\mathrm{L} \mathrm{ha}^{-1}$, disminuye las poblaciones de Spodoptera y Elasmopalpus, en épocas oportunas de control. Los producto a base de bacterias y hongos, como New bt (Bacillus thurigiensis) y Custom GP (Tricoderma harzianum) tiene una 
acción lenta de control, observándose mejor control sobre los cuatro días posteriores a la aplicación. El uso de T. harzianum, dentro de un programa de manejo de plagas, no garantiza un adecuado control dentro de las 96 horas posteriores a la aplicación.

\section{References:}

1. Betancourt, C. (1999). Guía de insectos y ácaros de importancia agrícola y forestal en Uruguay. Universidad de Montevideo. Editorial Universitaria. Montevideo, Uruguay. 125p.

2. Betancourt, C. \& Scatoni, I. (1999). Guía de insectos y ácaros de importancia agrícola y forestal en Argentina. Universidad Central de Buenos Aires. Editorial Universitaria. Buenos Aires, Argentina. $125 p$.

3. Biotecdor (2014). "NP-600, insecticida orgánico". In EDIFARM. Vademécum Agrícola 2014, 15ta edición. Editorial Poligráfica, Guayaquil-Ecuador. pp 176-175.

4. Centro de Investigación de Agricultura Tropical-CIAT. (1998). Micro Lepidóptera venezolana I. Bol. Revista Entomología Venezolana. 10:1-36p."

5. Ecuaquimica (2014). "Híbrido DK-1040”. In EDIFARM. Vademécum Agrícola 2014, 15ta edición. Editorial Poligráfica, Guayaquil-Ecuador. pp 267-268.

6. Ecuaquimica (2014). “Neem $X$, insecticida”. In EDIFARM. Vademécum Agrícola 2014, 15ta edición. Editorial Poligráfica, Guayaquil-Ecuador. pp 132-133.

7. FAO (2012). Estudio FAO sobre plagas en América latina. Roma. Revista Investigación y tecnología. 14:1-13.

8. Guzmán, GI. \& Alonso, AM. (2001). Manejo de malezas (flora espontánea en agricultura ecológica). Hoja Divulgativa 4.6/01. Dirección General de Investigación y formación Agraria. Barcelona, España. 19p.

9. Hilje, L. (1993). Efecto del clorpirifos sobre cogollero, en la zona del valle de San Juan. Costa Rica. Tesis Máster Ciencias. CATIE. P 4954.

10. INAHMI - Instituto Nacional de Hidrología y Meteorología (2016). Información climática para Ecuador: Año 2015. Informe Técnico anual. Quito, EC. Disponible en: http://www.inahmi.gob.ec/estamet.mediaanual.pdf

11. INEC- SINAGAP (Sistema Nacional de Gestión Agropecuaria) (2014). Zonificación agroecológica económica del cultivo de maíz duro (Zea mayz) en el ecuador. Resumen Técnico, PDF. Quito, EC. $14 \mathrm{p}$. 
12. Instituto Nacional Autónomo de Investigaciones AgropecuariasINIAP (2012). Tecnología disponible para la problemática entomológica en el litoral. Boletín técnico 89. EEB. pp 6-9.

13. Instituto Nacional Autónomo de Investigaciones AgropecuariasINIAP (2015). Plagas del maíz en el Ecuador. Boletín técnico 47. EEP. pp 4-8.

14. Khamby, B.; Batty, D.; Cahill, M. \& Simmons, M. (1991). Characterization and biological activity of naphtoquinones from Calceolaria andina. Agri Food-chem. 47(2):770-775.

15. Monzón, G. (2000). Producción y usos de hongos entomopatógenos para el control de plagas agrícolas. Managua-Nicaragua. Universidad Agraria de Nicaragua. 49p.

16. Navarro, R. (2000). Plagas del algodonero en Venezuela. Estado de Aragón. Universidad de Zulia. Editorial Universitaria. pp 4-9.

17. Negrete, F. \& Morales, J. (2003). Control orgánico de Spodoptera frugiperda, mediante insecticidas botánicos. Universidad de Sinu. Editorial Universitaria. Colombia. 115p.

18. Ortega, A. (1987). Insectos nocivos del maíz: Una guía para su identificación en el campo. Editorial Limusa. D.F. México. p 28-31.

19. Paliz, J. \& Mendoza J. (1985). Plagas del maíz en el litoral ecuatoriano, sus características y control. Instituto Nacional Autónomo de Investigaciones Agropecuarias-INIAP. Boletín Técnico $\mathrm{n}^{\circ} 12$. pp. 11-17

20. Quijije, R. (2016). Manejo integrado de plagas y enfermedades en la zona central del litoral ecuatoriano. Instituto Nacional Autónomo de Investigaciones Agropecuarias-INIAP. Boletin 14, Pichilingue, EC. pp. 1-11

21. Roberts, D. (1999). Wold picture of biological Control $f$ insect by fungi. Empresa de pesquisa agropecuaria-EMBRAPA. Brasil. pp 89100.

22. Suquilanda, M. (2013). Agricultura orgánica en el Ecuador. AGRIANDES.-QUITO. pp 45.55. 